\title{
Parametric Branch and Bound
}

\author{
FRED GLOVER
}

University of Colorado

(Received September 1977)

\begin{abstract}
The paper describes a procedure for mixed integer programming that allows branches to be imposed 'by degrees', which can subsequently be revised or weeded out according to their relative influence. It is an adaptive approach in which the branch and bound tree can be manipulated and restructured. The approach also yields measures of the costs of imposing the branches that lead to integer solutions, thus providing a built-in form of sensitivity analysis for evaluating the effect of integer restrictions.
\end{abstract}

\section{INTRODUCTION}

THIS paper introduces a parametric branch and bound $(B \& B)$ procedure that has greater flexibility than ordinary B\&B. Once a branch is taken in ordinary $\mathbf{B} \& \mathbf{B}$, it is largely irrevocable-i.e. all descendant branches must inherit the limitations imposed by their predecessors. In parametric $\mathbf{B} \& \mathbf{B}$, a descendant branch may partly or wholly undo an antecedent branch. Moreover, once a feasible mixed integer programming (MIP) solution is obtained, then the 'actual' branches that achieved this solution can be identified by weeding out uninfluential branches created during the solution process.

The strategy underlying parametric B\&B is to incorporate variables and constraints into the objective function in a manner resembling 'multi-objective' or 'goal programming' approaches $[1,2,9]$. The process is especially direct for 0-1 MIP problems, which do not require the creation of additional variables. However, even in the general case, all calculations can be carried out relative to a compact basis that is the same size as for the original problem.

The use of weighted variables and constraints in the objective function also bears a connection to 'Lagrangean' approaches $[3,6$, 10]. However, in contrast with Lagrangean techniques, the weights are not designed to solve a dual problem, but rather entirely over- shoot dual feasibility. The process may be viewed as that of constructing tentative duals (at least implicitly), guided by considerations relevant to the $B \& B$ setting. Also, in contrast to standard $B \& B$ and its exploitation of duality, parametric branches are conveniently handled by postoptimizing with the primal simplex method, whereas ordinary branches are often preferably handled by postoptimizing with the dual simplex method. Instead of imposing a branch either fully or not at all, parametric B\&B allows one to impose branches 'by degrees'. This is extremely important for enabling branching alternatives to be carefully analyzed - and revised, if desirable-at later stages of the tree.

By its nature, parametric B\&B yields information about the cost of imposing branches that lead to integer solutions. This information can then be used in a sensitivity analysis for evaluating the significance of integer restrictions. This type of analysis can also be coupled with an approach that attaches penalties to deviations from constraints. Such an approach yields a combined sensitivity analysis characterizing the influence of integer assignments on problem constraints.

\section{PARAMETRIC B\&B FOR 0-1 PROBLEMS}

The basic ideas of parametric B\&B are relatively straightforward for 0-1 MIP problems, 
and we first examine them in this setting. For our purposes, the 0-1 problem will be written

$$
\begin{aligned}
& \text { Minimize } x_{0}=\sum_{k \in \mathbb{N}} c_{j} x_{j} \\
& \sum_{i} a_{i j} x_{j} \leq b_{i} \\
& x_{j} \geq 0 \\
& 1 \geq x_{j} \geq 0 \\
& x_{1} \\
& \begin{array}{rr} 
& i \in M \\
& j \in M \\
& j \in J \\
\text { integer } & j \in J
\end{array}
\end{aligned}
$$

where $J$ is the index set of the integer variables and is a subset of $N$. In our discussion of $0^{-1}$ problems, we will confine ourselves to elaborating the principal ideas of parametric $\mathbf{B} \& \mathbf{B}$, together with illustrating the more rudimentary types of considerations. In fact, most of the implementation aspects of the $0-1$ case require no commentary other than to indicate the relevant decision alternatives. Subsequently, more advanced aspects of implementation will be introduced for the general MIP case, and then linked to the 'sensitivity analysis' framework.

In the 0-1 setting, consider any two disjoint subsets $J_{0}$ and $J_{1}$ of $J$ and the 'contrived' objective function

$$
\text { Minimize } z_{0}=d_{0}+\sum_{k J_{0}} d_{j} x_{j}-\sum_{k j} d_{j} x_{j}
$$

where all of the $d_{j}$ are positive, and where

$$
d_{0}=\sum_{k J_{1}} d_{j}
$$

By the form of $z_{0}$, if there exists a feasible solution to the LP problem (2-1)-(2-4) that satisfies $x_{j}=0$ for $j \in J_{0}$ and $x_{j}=1$ for $j \in J_{1}$ then this will be an optimal solution to the LP problem in which (2-1') replaces (2-1). (In fact, this assignment of values to the $x_{j}$ for $j \in J_{0} U J_{1}$ is uniquely optimal when feasible, and hence must occur at an LP extreme point. Note that this gives a rather simple proof of the fact that feasible integer assignments are unique extreme points of the pure $0-1$ problem and occur at one or more extreme points of the mixed $0-1$ problem.)

In addition, by the definition of $d_{0}$, it follows that $z_{0} \geq 0$ for all feasible LP solutions, and $z_{0}=0$ only for a solution that yields the assignment $x_{j}=0$ for $j \in J_{0}$ and $x_{j}=1$ for $j \epsilon J_{1}$. Consequently, the creation of a composite objective function

$$
\text { Minimize } u_{0}=x_{0}+z_{0}
$$

assures by the non-negativity of $z_{0}$ that

$$
\operatorname{Min} u_{0} \geq \operatorname{Min} x_{0} \text {. }
$$

Drawing on the fact that the constraint $z_{0}=0$ is equivalent to the 'partial assignment' $x_{j}=0$, $j \in J_{0}$ and $x_{j}=1, j \in J_{1}$ we may use the objective $\left(2-1^{\prime \prime}\right)$ as a device to compel this partial assignment to hold. Clearly, if the parameters $d_{j}$ are selected large enough, then $z_{0}=0$ must result provided the corresponding partial assignment is feasible.

Handling the composite objective function in parametric $B \dot{\&} B$

The first stage of the parametric B\&B approach for $0-1$ problems utilizes these ideas to influence the creation of partial assignments through manipulation of the parameters $d_{j}$. However, instead of assigning these parameters preemptively large values, more moderate values are assigned and then monitored in order to determine interactive effects relevant to the B\&B setting. By the familiar Lagrangean type of argument, we may observe

$$
\begin{aligned}
\text { Min }\left\{x_{0} \text { subject to } z_{0}=0\right\} \\
=\left\{\text { Min } u_{0} \text { subject to } z_{0}=0\right\} \geq \operatorname{Min} u_{0}
\end{aligned}
$$

and hence the composite objective function $\left(2-1^{\prime \prime}\right)$ always yields a lower bound on the ordinary $\mathbf{B} \& B$ objective (in which $z_{0}=0$ is explicitly imposed). This type of bound information can be used for fathoming in a manner resembling that of the ordinary $\mathbf{B} \& \mathbf{B}$ approach. We will discuss the way to accommodate fathomed alternatives in the parametric setting after introducing the notion of a 'parametric branch'.

\section{0-1 Parametric branching}

The branch step for the 0-1 parametric approach is a 'tentative' operation that either becomes consolidated or revised on the basis of information subsequently generated. Quite simply, if the branch corresponds to the assignment $x_{r}=0$ then the current objective $u_{0}$ is updated to become $u_{0}+d_{r} x_{r}$ and if the branch corresponds to the assignment $x_{r}=1$ then $u_{0}$ is updated to become $u_{0}+d_{r}\left(1-x_{r}\right)$ or equivalently $u_{0}+d_{r} x_{r}^{\prime}$ where $x_{r}^{\prime}$ is the slack variable for the inequality $x_{r} \leq 1$. The weight $d_{r}$ is selected so that the updated representation of $u_{0}$ is dual infeasible, and therefore, allows reoptimization with the primal simplex method.

To illustrate, suppose the current LP representations of $u_{0}$ and $x_{r}$ are given by

$$
\begin{gathered}
u_{0}=12 \frac{1}{4}+5 \frac{1}{2} x_{3}+1 \frac{1}{4} x_{4}+4 x_{5} . \\
x_{r}=\frac{1}{4}-2 x_{3}+x_{4}+\frac{3}{4} x_{5} .
\end{gathered}
$$


Then for the branch $x_{r}=0$ any value of $d_{r}$ satisfying $d_{r}>5 \frac{1}{2} / 2$ will cause the new objective $u_{0}+d_{r} x_{r}$ to be dual infeasible. (The ratio $5 \frac{1}{2} / 2$ is exactly the pivot ratio for the dual simplex method that would be identified if one were to introduce the constraint $x_{r} \leq 0$.) Thus, for example, selecting $d_{r}=3$, we obtain the new $u_{0}$ objective

$$
u_{0}=13-\frac{1}{2} x_{3}+4 \frac{1}{4} x_{4}+6 \frac{1}{4} x_{5} .
$$

The negative coefficient for $x_{3}$ of course signals that re-optimization may now be undertaken with the primal simplex method.

Alternatively, for the branch $x_{r}=1$ a value of $d_{r}$ satisfying $d_{r}>1 \frac{1}{4} / 1$ will succeed in establishing dual infeasibility for the objective $u_{0}+d_{r} x_{r}^{\prime}$ where $x_{r}^{\prime}=1-x_{r}$. (Here $1 \frac{1}{4} / 1$ is the pivot ratio for the dual simplex method relative to the constraint $x_{r} \geq 1$.) Thus, for example, selecting $d_{r}=2$ we obtain the new objective

$$
u_{0}=1 \frac{3}{4}+9 \frac{1}{2} x_{3}-\frac{3}{4} x_{4}+2 \frac{1}{2} x_{5} .
$$

Values of $d_{r}$ such as those selected in these examples may not be sufficiently large to insure that the branches for $x_{r}=0$ and $x_{r}=1$ will utlimately be enforced. However, as previously noted, the procedure does not seek preemptive values but rather seeks to analyze the consequences of more moderate values. (In this connection, it is easily established that if a dual pivot on the constraint $x_{r} \leq 0$ or $x_{r} \geq 1$ would achieve primal feasibility in one step; then any value of $d_{r}$ that exceeds the dual pivot ratio will achieve exactly this same solution in one primal iteration.)

This extremely simple type of 'local' implementation step for $0-1$ parametric $B \& B$ has a direct analog in a variety of standard 'parametric' approaches for ordinary linear programming. The crucial aspect in the $B \& B$ setting is the way in which this step is used-i.e. the manner in which the parametric branches are processed to yield information and branching alternatives not available to ordinary $\mathbf{B} \& \mathbf{B}$. Thus, in particular, parametric branches of the form just illustrated are not regarded as ironclad impositions nor are they initially assigned a sequential ranking. Indeed, it may well occur at a subsequent step that an 'earlier' branch will be discovered to be superfluous in terms of other more influential branches. We discuss this aspect of the approach next.

\section{Revised and augmented parametric branches}

Branches that are currently uninfluential can easily be singled out by the parametric B\&B procedure as follows. Suppose the updated LP representation of $u_{0}$ gives $x_{r}$ or $x_{r}^{\prime}$ (depending on which of the two variables was previously assigned a weight) a coefficient that equals or exceeds $d_{r}$. (The implication, of course, is that $x_{r}$ or $x_{r}^{\prime}$ is currently nonbasic.) Then, reducing this coefficient by $d_{r}$ still leaves the objective dual feasible. This means that the parametric branch can be eliminated without changing the current LP solution.

The step of weeding out uninfluential branches (i.e. those subject to elimination) can be postponed until a feasible $0-1$ solution is obtained. Then, all variables whose parameter values can be reduced to zero may be excluded from the branching category. (An exception occurs for branches that are antecedents of a compulsory branch, as described in the next section.) The remaining variables and parametric branches may further be ranked, for example, according to the magnitude of the $d_{r}$ values that are required to make the current solution optimal. In this fashion, the 'actual' branches of the B\&B process, and their sequence, are decided upon at a stage in which the true significance of these branches can more accurately be assessed.

Another means for evaluating the relative influence or significance of a branch occurs when a branching variable 'resists' its weight and becomes basic. The decision must then be whether to increase or decrease $d_{r}$. (The latter may be a compounded step that both decreases $d_{r}$ to 0 for the current parametric branch and then assigns $d_{r}$ a positive value for the alternative branch.) By means of such decisions, earlier parametric branches may either be revised or reinforced.

The technical aspects of implementing these ideas are relatively simple, and hence, we confine ourselves to the task of discussing only the more prominent strategic considerations, deferring the particulars of implementation to the general MIP case, where they are more critical.

\section{Compulsory branches and sequences}

The minimization of $u_{0}$ as noted earlier provides a lower bound on the optimum value of $x_{0}$ subject to the partial assignment associated 
with $z_{0}=0$. Whenever this bound equals or exceeds the value of $x_{0}$ for the best MIP solution currently known (which automatically occurs when the current LP solution in fact provides this best MIP solution), then all further continuations of the partial assignment are identified as unproductive in the usual B\&B sense. This immediately provides the option of 'back-tracking'-i.e. of deciding which parametric branch underlying the current LP solution should be considered the "last branch'. The observation that the determination of the last branch can be deferred to yield a more flexible variant of the LIFO procedure was first made by Tuan [11]. In the current approach, this determination can be based, for example, on the current $d_{j}$ values (after weeding out uninfluential branches). Thus, in this fashion, a sequential ordering is gradually imposed on the branches.

Having identified a last branch, the alternative branch is now imposed as compulsory. This means that the method does not allow this branch to be reversed or discarded (following the usual backtracking rules), until the current partial assignment is fathomed. In particular, as soon as a compulsory branch is identified, all other parametric branches that are currently in force (i.e. all those for which $d_{j}$ is currently positive) must be considered as prior to the compulsory branch. This does not impose any particular sequence on these prior branches (until backtracking again compels one to be identified as a 'last member'), but does impose induced bounds on the parameters $d_{j}$. That is, these positive $d_{j}$ coefficients cannot be reduced below their current values as long as the compulsory branch remains in effect.

There are, however, three important exceptions to the strict maintenance of induced bounds. The first and obvious exception occurs by deciding upon some partial sequence for the parametric branches and employing a tree search rule that jumps back over some of the 'later' branches of this sequence. Then, the induced bounds can be disregarded for the branches thus bypassed. (The same remark holds for branches that are released or reversed as a result of carrying out a backtracking step with the LIFO rule.) The second exception occurs by redeciding the status of the branch that has been designated compulsory. That is, a compulsory branch may be revised, if the reconfigured objective $u_{0}$ that results after a series of additional iterations makes this appear eminently desirable. (Such a process must, of course, be sufficiently systematized to guard against circularity.) Finally, just as compulsory branches may be imposed as standard branches rather than parametric branches (as by the use of pre-emptive weights), so may the identified antecedents of the compulsory branches be imposed in the standard fashion. This imposition, however, does not carry with it an implied sequence for the antecedents themselves. Further, greater flexibility is achieved by monitoring the values of parameters that would suffice to maintain the imposed branches, thereby retaining the option of amending the status of these branches at subsequent stages.

\section{PARAMETRIC B\&B FOR GENERAL MIP PROBLEMS}

Many of the same strategic notions discussed in the preceding section carry over to the general MIP problem. However, there is a very significant difference between the general problem and the 0-1 problem. Specifically, in the general case, there may not exist weights that will cause all of the branching inequalities to be satisfied. Moreover, if such weights exist, they may be very hard to find, or cause some inequalities to be unnecessarily over-satisfied, thus failing to identify integer solutions. We now examine the special techniques that permit these difficulties to be overcome and allow the general approach to be implemented successfully.

Branching schemes for the general MIP problem may be viewed as the successive imposition of upper and lower bounds on the problem variables. Thus, at any particular stage, the integer variables are governed by restrictions of the form

$$
U_{j} \geq x_{j} \geq L_{j} \quad j \in J
$$

where $U_{j}$ and $L_{j}$ may represent original bounds on $x_{j}$ or those currently inherited by branching. The general parametric approach seeks to incorporate such bound restrictions into the objective function in a manner that will enable the same types of evaluations and manipulations that are possible in the $0-1$ setting. 
This is accomplished by introducing nonnegative variables $z_{j}$ and the inequalities

$$
U_{j}+z_{j} \geq x_{j} \geq L_{j}-z_{j} \quad j \in J .
$$

Thereupon, the $z_{j}$ variables are incorporated into the minimization of $x_{0}$ to create the composite objective function

$$
\text { Minimize } u_{0}=\sum_{j \in N} c_{j} x_{j}+\sum_{j \in J} d_{j} z_{j} .
$$

It is clear that for the weights $d_{j}$ sufficiently large all of the $z_{j}$ will be 0 and (3-2) will reduce to (3-1)-provided, of course, that (3-1) is consistent with the original problem constraints. Also, for $d_{j}>0$ we have

and

$$
\operatorname{Min} u_{0} \geq \operatorname{Min} x_{0}
$$

$$
\begin{array}{r}
\text { Min }\left\{x_{0} \text { subject to }(3-1)\right\} \\
=\operatorname{Min}\left\{u_{0} \text { subject to }(3-1)\right\} \geq \operatorname{Min} u_{0} .
\end{array}
$$

Thus, the same bound relationships hold in the general MIP settings as in the $0-1$ context upon introducing (3-2) and (3-3).

The problem at hand is how to manage the system (3-2) and (3-3) effectively. This is extremely important because otherwise, the introduction of (3-2) and (3-3) doubles the number of integer variables and adds a corresponding number of new constraints. The following approach for dealing with this problem constitutes an adaptation of procedures for 'weighted deviation' problems introduced in [7].

Let $s_{j}^{\prime}$ and $s_{j}^{\prime \prime}$ denote slack variables for the inequalities of (3-2), yielding the equations

$$
\begin{aligned}
x_{j}-z_{j}+s_{j}^{\prime} & =U_{j}, \\
-x_{j}-z_{j}+s_{j}^{\prime \prime} & =-L_{j}
\end{aligned}
$$

Adding these two equations, we obtain

$$
-2 z_{j}+s_{j}^{\prime}+s_{j}^{\prime \prime}=U_{j}-L_{j}
$$

Equation (3-5) identifies a 'primal relationship' between the variables $z_{j}, s_{j}^{\prime}$ and $s_{j}^{\prime \prime}$. On the 'dual' side, let $u_{j}, v_{j}^{\prime}$ and $v_{j}^{\prime \prime}$ represent dual variables associated with the non-negativity restrictions on $z_{j} s_{j}^{\prime}$ and $s_{j}^{\prime \prime}$ respectively. (That is, the defining equations for $u_{j}, v_{j}^{\prime}$ and $v_{j}^{\prime \prime}$ are respectively given by the $z_{j}, s_{j}^{\prime}$ and $s_{j}^{\prime \prime}$ columns of the primal.) Then, since the coefficients of $z_{j}, s_{j}^{\prime}$ and $s_{j}^{\prime \prime}$ are $d_{j}, 0$ and 0 in (3-3), it follows from (3-4) that the relationship between the columns for $z_{j}, s_{j}^{\prime}$ and $s_{j}^{\prime \prime}$ can be summarized by

$$
u_{j}+v_{j}^{\prime}+v_{j}^{\prime \prime}=d_{j}
$$

This relationship, in conjunction with (3-5), provides the key to implementing the general paramatric B\&B approach without modifying the size of the LP tableau. In particular, it is readily established that at least one of the variables $z_{j}, s_{j}^{\prime}$ and $s_{j}^{\prime \prime}$ must be basic and at least one must be nonbasic.

Consequently, only one of the three primal variables need be explicitly included in the tableau at any given time, whereupon the form of the other variables is always known from (3-5) and (3-6). Similarly, $x_{j}$ need not be included in the tableau since it is always capable of being recovered from an equation of (3-4). The fundamental relationships of the approach as they apply in the present setting can be summarized as follows. (Formal proofs of these relationships are omitted, since their derivation is a direct consequence of (3-5) and (3-6).)

(R.0)-Of the two variables (from the group $z_{j}, s_{j}^{\prime}$ and $\left.s_{j}^{\prime \prime}\right)$ that are not explicitly included in the LP tableau, one is currently basic and one is currently nonbasic.

\section{(R.1)-If the explicit variable is nonbasic:}

(a) The tableau row for the implicit basic variable is precisely the primal equation (3-5).

(b) The tableau column for the implicit nonbasic variable is the negative of the column for the explicit variable, except that the objective function coefficient for the implicit variable is $d_{j}$ minus the objective function coefficient of the explicit variable, and the column coefficient that corresponds to the implicit basic variable is as given in (a).

\section{(R.2)-If the explicit variable is basic:}

(a) The tableau column for the implicit nonbasic variable is precisely the dual equation (3-6). Thus, the objective function coefficient is $d_{j}$ and the column has unit coefficients in the positions corresponding to its two companion basic variables, with 0 's elsewhere.

(b) The tableau row for the implicit basic variable is obtained by substituting the current expression for the explicit basic variable in (3-5) and giving the implicit nonbasic variable a unit coefficient [as in (a)].

We can now give the rules for implementing the parametric B\&B approach that result from these relationships. After stating these rules, we will illustrate their use by numerical example.

1. The initial step solves the LP problem without the additional variables and constraints of (3-4). 
2. The parametric branching step introduces the restrictions (3-4) in a tableau for which $x_{j}$ is basic and does not satisfy $U_{j} \geq x_{j} \geq L_{j}$.

(a) Identify the equation of (3-4) which implies $z_{j}>0$ for $s_{j}^{\prime}$ and $s_{j}^{\prime \prime}$ nonnegative (i.e. the equation corresponding to the 'violated bound');

(b) Omit the slack variable $s_{j}^{\prime}$ or $s_{j}^{\prime \prime}$ from the equation identified in (a), and use this reduced equation to replace $x_{j}$ with $z_{j}$ by direct substitution. The omitted slack variable is the implicit nonbasic variable, and slack variable of the other equation of (3-4) is the implicit basic variable. (These implicit variables are irrelevant at this point). The variable $z_{j}$ is now basic.

3. The next component step of parametric branching is to create (or increase) the weight $d_{j}$ for a selected variable $z_{j}$ that is currently basic. To do this, add $d_{j}^{\prime}$ times the current $z_{j}$ equation to the objective function equation, where $d_{j}^{\prime}$ denotes the increment in $d_{j}$, selected large enough to require re-optimization with the primal method. (The effect of this step on any currently implicit nonbasic variables is immediately given in relation (R.1)(b), since none of the implicit nonbasic variables governed by (R.2)(a) are affected except for the companion of $z_{j}$ whose coefficient is always $d_{j}$ )

4. An arbitrary iteration of the primal method can be implemented by the following observations:

(a) An implicit nonbasic variable provides an eligible pivot column if and only if its explicit companion is nonbasic and has a coefficient exceeding $d_{j}$ (as a result of relations (R.2)(a) and (R.1)(b)).

(b) If an implicit nonbasic variable is selected for the pivot column, then it can replace the previous explicit variable, which now becomes implicit.

(c) No implicit basic variable need be considered in determining the pivot row unless both slack variables $s_{j}^{\prime}$ and $s_{j}^{\prime \prime}$ are basic. If an implicit basic variable provides the pivot row, it replaces the currently explicit basic variable as the new explicit variable.

Condition $4(c)$ is a direct consequence of (3-5) and implies that once a slack variable of (3-4) first becomes implicitly basic, it remains irrelevant unless the other slack becomes basic-or until a new branch is introduced by tightening one of the $U_{j}$ or $L_{j}$ values. Branching can occur only if both of the slacks $s_{j}^{\prime}$ and $s_{j}^{\prime \prime}$ are basic and positive (since otherwise $x_{j}$ would be assigned an integer value or lie outside one of its provisional current bounds) and thus, in this case, one can simply jettison all three of the $s_{j}^{\prime}, s_{j}^{\prime \prime}$ and $z_{j}$ variables [after recovering $x_{j}$ from (3-4)] and start again with Step 2.

In accordance with these observations, if either $U_{j}$ or $L_{j}$ is a 'true' bound for $x_{j}$, then this bound can be rigidly enforced simply by rendering $z_{j}$ an ineligible pivot column (e.g. by increasing $d_{j}$ ) whenever the slack variable associated with the true bound is nonbasic. (If $z_{j}$ becomes basic when the alternative slack is nonbasic, (3-5) implies that $x_{j}$ must satisfy its appropriate bound, and condition 4(c) assures that the critical slack will not become nonbasic unless $z_{j}$ is nonbasic.)

We now illustrate these considerations with the following numerical example.

\section{Example}

Consider the MIP problem represented by the following 'condensed' tableau format, where $x_{1}$ and $x_{2}$ are the integer variables.

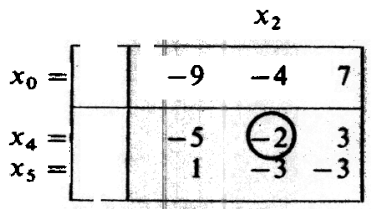

The problem constraint equations are read directly from the tableau (e.g. $x_{4}=7-5 x_{1}-$ $2 x_{2}+3 x_{3}$ ). Pivoting on the circled element with the primal simplex method yields the optimal LP tableau

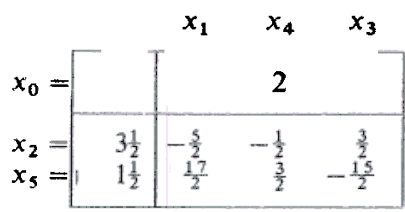

Suppose now that we wish to branch parametrically on the inequality $x_{2} \leq 3$ by Step 2 of the preceding rules, thus obtaining $U_{2}=3$ and $L_{2}=0$ (where $L_{2}=0$ represents a true lower bound). The first equation of (3-4) implies $z_{2}>0$ and yields $z_{2}=x_{x}-U_{2}$ upon omitting 
the slack variable $s_{2}^{\prime}$. Thus, the tableau equation for $z_{2}$ is given by

$$
z_{2}=x_{2}-3=\begin{array}{|l|lll|}
x_{1} & x_{4} & x_{3} \\
\hline \frac{1}{2} & -\frac{3}{2} & -\frac{1}{2} & \frac{3}{2} \\
\hline
\end{array}
$$

Next, by Step 3, we add a sufficient multiple of this equation to the objective equation to create dual infeasibility. Any value exceeding the 'dual pivot ratio' $1 /(5 / 2)$ will do, and we select a multiple of 1 to give $u_{0}=x_{0}+1 z_{2}$ or

$$
\begin{array}{rlrr} 
& x_{1} & x_{4} & x_{3} \\
\hline & -13 t & -\frac{3}{2} & \frac{3}{2} \\
\hline
\end{array}
$$

Thus, putting Steps 2 and 3 together (replacing the $x_{2}$ equation by the $z_{2}$ equation and replacing $x_{0}$ by $u_{0}$ ) we obtain the tableau

\begin{tabular}{l|r|rrr|} 
& $x_{1}$ & \multicolumn{1}{c}{$x_{4}$} & \multicolumn{1}{c}{$x_{3}$} \\
$u_{0}=$ & $-13 \frac{1}{2}$ & $-\frac{3}{2}$ & $\frac{3}{2}$ & $\frac{5}{2}$ \\
$z_{2}=$ & $\frac{1}{2}$ & $-\frac{3}{2}$ & $-\frac{1}{2}$ & $\frac{3}{2}$ \\
$x_{5}=$ & $\frac{1}{2}$ & $\frac{17}{2}$ & $\frac{3}{2}$ & $-\frac{15}{2}$ \\
\hline
\end{tabular}

The primal simplex method now pivots on the

\begin{tabular}{|c|c|c|c|c|}
\hline & & $z_{2}$ & $x_{4}$ & $x_{3}$ \\
\hline & & $\frac{3}{5}$ & $\frac{2}{5}$ & $\frac{8}{5}$ \\
\hline & $\begin{array}{l}\frac{1}{3} \\
\frac{16}{5}\end{array}$ & $\begin{array}{l}-\frac{2}{3} \\
-\frac{17}{3}\end{array}$ & $\begin{array}{l}-\frac{1}{5} \\
-\frac{1}{5}\end{array}$ & $\begin{array}{r}\frac{3}{5} \\
-4^{2}\end{array}$ \\
\hline
\end{tabular}
circled element to obtain

Since optimality is re-established in a single pivot, this is the same tableau that would have been obtained by a 'dual' pivot on the constraint $x_{2} \leq 3$ (except for the $z_{2}$ column). With $z_{2}$ and $s_{2}^{\prime}$ both nonbasic (the first explicitly, the second implicitly), we may infer that the current value of $x_{2}$ is 3 (as seen by (3-4) with $U_{2}=3$ ). Consequently, at the present stage the parametric branch has achieved the same effect as an ordinary branch, although we do not assign the branch a sequential 'slot' and leave it open to subsequent revision.

Suppose that $x_{1} \geq 1$ is selected to provide the next parametric branch. By Step 2, the relevant expression for $z_{1}$ is $z_{1}=L_{1}-x_{1}=$ $1-x_{1}$ giving the tableau representation

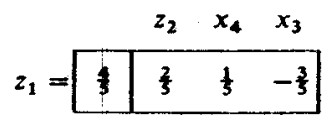

By Step 3, we again seek to add a sufficient multiple of $z_{1}$ to the current objective function to produce a dual infeasibility. Since the implicit nonbasic variable $s_{2}^{\prime}$ has a coefficient which is the negative of the explicit $z_{2}$ coefficient (i.e. $-\frac{2}{5}$ ), we must take the effect of $s_{2}^{\prime}$ into account in the manner noted parenthetically in Step 3. By relation (R.1)(b), the current objective function coefficient of $z_{2}$ is $1-\frac{3}{5}=\frac{2}{5}$. This yields the 'expanded' current $u_{0}$ and $z_{1}$ rows as follows

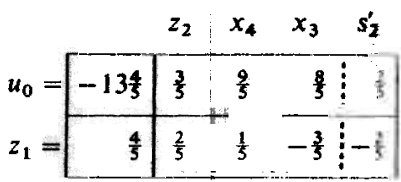

From this, we see that a multiple of 2 suffices to produce dual infeasibility thereby giving the new objective function representation

$$
\begin{array}{ll|l|lll|} 
& z_{2} & x_{4} & x_{3} & s_{2}^{\prime} \\
\hline u_{0}=-12 \frac{1}{3} & \frac{7}{3} & \frac{4}{3} & \frac{2}{3} & -\frac{2}{3} \\
\hline
\end{array}
$$

Note that the 'expansion' used in this example was not really necessary since the fact that $z_{2}$ has a positive coefficient immediately implies that we consider the implicit variable instead of-rather than in addition to-the explicit variable.

Because $s_{2}^{\prime}$ gives the eligible pivot column, we replace $z_{2}$ by $s_{2}^{\prime}$ (so that $z_{2}$ now becomes implicit) to yield the tableau

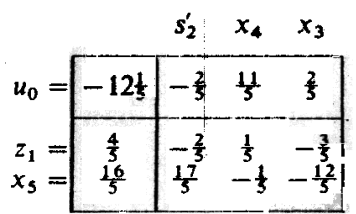

Re-optimization with the primal method gives

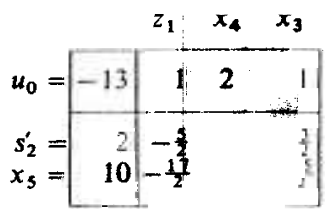

The variables of (3-4) associated with $x_{1}$ and $x_{2}$ have integer values in this tableau, and hence so do $x_{1}$ and $x_{2}$. Specifically, direct application of (3-4) yields $x_{1}=1$ and $x_{2}=1$ (noting that $s_{1}^{\prime \prime}$ and $z_{2}$ are implicitly nonbasic). Also, since $z_{1}$ and $z_{2}$ are both in the current 
basic solution, it follows that $u_{0}$ and $x_{0}$ have the same value.

We now analyze the parametric branches that provided this current integer solution. By relation (R.2)(a), the objective function coefficient of $z_{2}$ is $d_{2}$. Thus, reducing the coefficient of $z_{2}$ by $d_{2}$ (which effectively removes $z_{2}$ as a weighted variable in the objective function) still yields the same LP optimum. By our earlier observations for the $0-1$ case, this means that the branching restriction that gave rise to $z_{2}$ is currently 'uninfluential', and thus we can shrink the B\&B tree to the single restriction $x_{1} \geq 1$.

At this stage, since the current solution is locally optimal, it is unproductive to proceed with the present line of branching. Thus $x_{1} \geq 1$ is replaced by the compulsory branch $x_{1} \leq 0$, and the solution process continues.

\section{PARAMETRIC B\&B AND MIP SENSITIVITY ANALYSIS}

Two types of sensitivity analysis are available with parametric $B \& B$ as illustrated in the preceding section. The first involves identifying the 'cost' of branches that lead to particular integer solutions. For example, in the earlier numerical example, it was noted that essentially no cost attached to branching on $x_{2}$ to obtain the first integer solution. (Consequently, the integer restriction for $x_{2}$ was judged 'conditionally superfluous' and the branch was discarded in this instance.) Further, the tableau that yields the integer solution assigns $z_{1}$ an objective function coefficient of 1 , which means that $d_{1}$ can be reduced from its present value of 2 to $2-1=1$ without altering the LP optimum. This places the cost of the $x_{1}$ branch underlying this solution at 1 , which provides a measure of the relative significance of this branch and the integer restriction on $x_{1}$ in the current solution. Specifically, we can conclude that relaxing this branch will result in at least 1 unit of improvement in the objective function for each unit of change in the value of $x_{1}$. Corresponding interpretations apply to situations in which multiple branches underlie an integer solution.

The second type of sensitivity analysis involves the explicit assignment of penalties to deviating from particular problem constraints.
Coupling this penalty procedure with parametric $B \& B$, the interactive effect of constraint deviations and parametric branches becomes susceptible to evaluation. For example, the updated cost of a variable representing a constraint violation identifies the net effective cost of imposing the constraint relative to a given integer solution. Thus, if the constraint is in fact violated at this solution, the $d_{j}$ values for the parametric branches not only reflect branching costs relative to the 'original' objective function but also relative to the penalty incurred from the violated constraint. This enables a more realistic form of problem solving in situations where constraint deviations may be tolerable at a cost. The entire analysis and solution process can be conveniently carried out by merging the compact basis procedures illustrated for the MIP problem with the more elaborate procedures of [7] to maintain the working tableau the same size as the original.

\section{REFERENCES}

1. Charnes A \& Cooper W (1961) Management Models and Industrial Applications of Linear Programming, vol. 1, John Wiley, New York.

2. Charnes A, CoOper W \& Ijiri U (1963) Breakeven budgeting and programming to goals. J. Acc. Res. 1(1).

3. Fisher ML \& SHAPIRo JF (1973) Constructive duality for discrete optimization. CMBSE Report 7321, Graduate School of Business, University of Chicago.

4. GASS SI \& SAATY TL (1955) The computational algorithm for the parametric objective function. Nav. Res. Logist. Q. 2, 39-45.

5. GEOFFRION AM (1966/67) Strictly concave parametric programming, Parts I and II. Mgmt Sci. 13(3), 244-253 and 13(5), 359-370.

6. GEOFfRION AM (1974) Lagrangean relaxation for integer programming. Mathl Progmg St. 2, 82-114.

7. GLOVER $F$ (1976) Linear programming for weighted deviation problems using compact basis techniques. MSRS 76-14, University of Colorado.

8. GLOVER F \& TANGEDAFL L. (1976) Dynamic strategies for branch and bound. Omega 4(5). 571-576.

9. JOHNSEN E (1968) Studies in multiobjective decision models. Suden Litteratur.

10. SHAPIRO JF (1971) Generalized lagrange multipliers in integer programming. Ops. Res. 19(1), 68-76.

11. TUAN NP (1971) A flexible tree search method for integer programming problems. Ops. Res. 19, 115-119.

ADDRESS FOR CORRESPONDENCE: Professor Fred Glover, College of Business Administration, University of Colorado, Boulder, CO 80309, USA. 